\title{
Effects of Built-In Polarization on InGaN-GaN Vertical-Cavity Surface-Emitting Lasers
}

\author{
Joachim Piprek, Robert Farrell, Steve DenBaars, and Shuji Nakamura
}

\begin{abstract}
We investigate the effect of built-in spontaneous and piezoelectric polarization on the internal device physics of current-injected GaN-based vertical-cavity surface-emitting lasers (VCSELs) with strained InGaN quantum wells. Advanced device simulation is applied to a previously manufactured device design featuring dielectric mirrors and an indium-tin-oxide current injection layer. Contrary to common perception, we show: 1) that only a small fraction of the built-in quantum-well polarization is screened at typical injection current densities and 2) that the polarization of the AlGaN electron stopper layer has a strong effect on the VCSEL threshold current which can be partly compensated for by higher p-doping.
\end{abstract}

Index Terms-Electron leakage, GaN-based light emitter, InGaN quantum well, numerical simulation, piezoelectric effect, polarization, vertical-cavity surface-emitting laser (VCSEL).

$\mathbf{P}$ IEZOELECTRIC and spontaneous polarization is known to be much stronger in c-plane GaN-based alloys than in other III-V compounds. Extensive experimental and theoretical work has been invested in this phenomenon, and nonlinear analytical approximations have been derived for calculating the built-in polarization [1]. Polarization strongly affects radiative recombination processes in strained $\mathrm{InGaN}$ quantum wells which are typically employed in GaN-based light-emitting devices [2]. The polarization-induced electrostatic field leads to a separation of electrons and holes within the quantum well and thereby to a reduction of the photon emission rate. However, for GaN-based lasers, polarization effects are usually considered less important because the high density of electrons and holes in the quantum wells is assumed to screen the built-in polarization charges [3].

Using advanced device simulation, we investigate the effects of polarization on the internal physics and the threshold current of InGaN-GaN laser diodes. Our software self-consistently combines wurtzite quantum well band structure calculations, radiative and nonradiative carrier recombination, carrier drift and diffusion, and optical mode computation [4]. More details of the model are described elsewhere [5]. Previously, we used a very similar model to study edge-emitting high-power InGaN-GaN lasers, demonstrating good agreement with measurements [6].

Here, we focus on InGaN-GaN vertical-cavity surface-emitting lasers (VCSELs), which are expected to exhibit several

Manuscript received June 28, 2005; revised September 15, 2005. This work was supported in part by the U.S. Department of Energy and by the Solid State Lighting and Display Center, University of California, Santa Barbara.

The authors are with the Materials Department, University of California, Santa Barbara, CA 93106 USA (e-mail: piprek@ieee.org).

Digital Object Identifier 10.1109/LPT.2005.860045

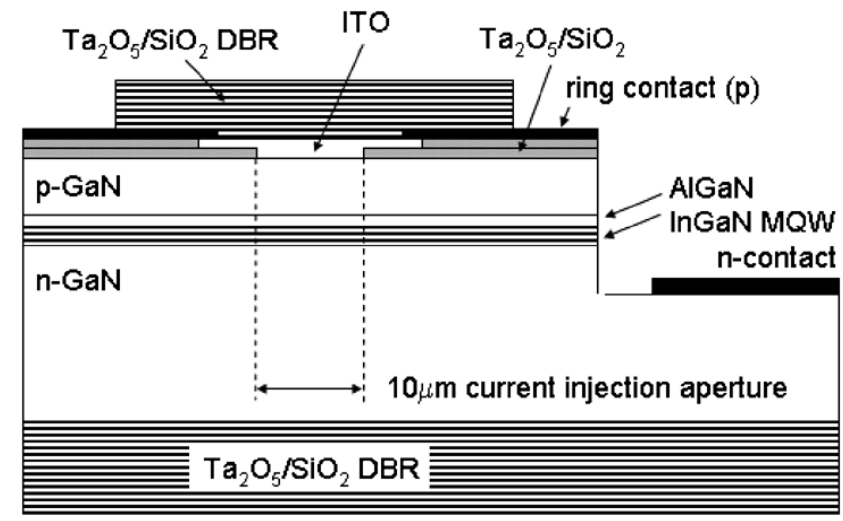

Fig. 1. Schematic VCSEL design.

advantages over their edge-emitting counterparts, including lower manufacturing costs, circular output beam, and longer lifetime. In contrast to the success of GaAs-based VCSELs in recent years, GaN-based VCSELs face significant challenges and only optically pumped devices have been reported thus far [7]. Our theoretical study is based on an injection-type 405-nm VCSEL that was designed, fabricated, and characterized earlier [8]. Fig. 1 shows the schematic design of this device which was grown by metal-organic chemical-vapor deposition (MOCVD) on sapphire. The multiquantum well (MQW) active region consists of five 4-nm-thick $\operatorname{In}_{0.1} \mathrm{Ga}_{0.9} \mathrm{~N}$ quantum wells and 8-nm-wide Si-doped $\mathrm{In}_{0.035} \mathrm{Ga}_{0.965} \mathrm{~N}$ barriers, and it is covered by a $20-\mathrm{nm} \mathrm{p}-\mathrm{Al}_{0.18} \mathrm{Ga}_{0.82} \mathrm{~N}$ electron stopper layer to reduce electron leakage into the p-GaN spacer layer [9]. An indium-tin-oxide (ITO) p-contact layer is employed, which confines the current injection to a $10-\mu \mathrm{m}$ wide aperture. The top metal ring contact confines the optical mode to about a $12-\mu \mathrm{m}$ diameter. Eleven-period dielectric distributed Bragg reflectors (DBRs) are used on both sides, which exhibit a high reflectance above 99\%. Among other reasons, built-in polarization is considered one of the factors that prevented this device from lasing [10].

The built-in polarization is represented by fixed interface charges at every hetero interface within the device (Table I). Fig. 2 compares the MQW energy band diagrams as calculated with and without polarization charges, using the $\mathrm{AlGaN}$ hole density as a parameter. The polarization significantly deforms the energy band diagram. This is remarkable considering the relatively high injection current density of $j=10 \mathrm{kA} / \mathrm{cm}^{2}$ used in this simulation, which is more than triple the threshold current density of similar edge-emitting lasers [6]. Surprisingly, 
TABLE I

Built-in Polarization Charge Densities Calculated for DifFerent TYPES OF INTERFACES IN OUR DEVICE USING MODEL IN [1]

\begin{tabular}{|l|l|}
\hline Interface & Charge Density \\
\hline $\mathrm{GaN} / \mathrm{In}_{0.035} \mathrm{Ga}_{0.965} \mathrm{~N}$ & $-3.2 \times 10^{12} \mathrm{~cm}^{-2}$ \\
\hline $\mathrm{In}_{0.035} \mathrm{Ga}_{0.965} \mathrm{~N} / \mathrm{In}_{0.1} \mathrm{Ga}_{0.9} \mathrm{~N}$ & $-6.5 \times 10^{12} \mathrm{~cm}^{-2}$ \\
\hline $\mathrm{In}_{0.1} \mathrm{Ga}_{0.9} \mathrm{~N} / \mathrm{In}_{0.035} \mathrm{Ga}_{0.965} \mathrm{~N}$ & $+6.5 \times 10^{12} \mathrm{~cm}^{-2}$ \\
\hline $\mathrm{In}_{0.0 .35} \mathrm{Ga}_{0.96} \mathrm{~N} / \mathrm{Al}_{0.18} \mathrm{Ga}_{0.82} \mathrm{~N}$ & $+11 \times 10^{12} \mathrm{~cm}^{-2}$ \\
\hline $\mathrm{Al}_{0.18} \mathrm{Ga}_{0.82} \mathrm{~N} / \mathrm{GaN}$ & $-7.8 \times 10^{12} \mathrm{~cm}^{-2}$ \\
\hline
\end{tabular}

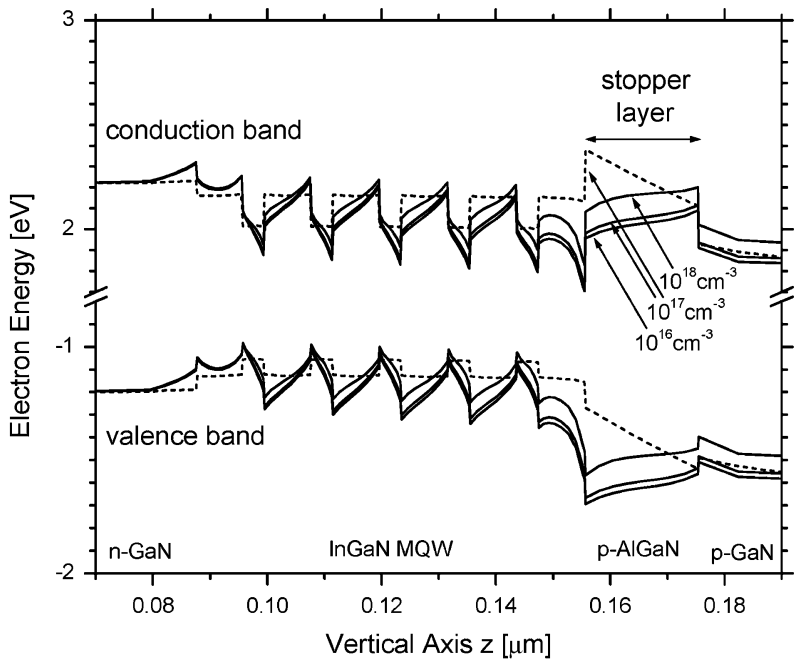

Fig. 2. Energy band diagram of the MQW active region at $10 \mathrm{kA} / \mathrm{cm}^{2}$ injection current density: dashed line is no polarization and solid line is full polarization (parameter: AlGaN hole density).

the built-in polarization field is not screened as is commonly assumed for laser operation.

In order to further evaluate this finding, we also plot carrier densities and net electrostatic field (Fig. 3). As expected, electrons and holes are clearly separated within the quantum wells due to the built-in field. The polarization charge densities at the MQW interfaces translate into a built-in quantum well field of $1.8 \mathrm{MV} / \mathrm{cm}$. The actual electrostatic field in Fig. 3 is less than $1 \mathrm{MV} / \mathrm{cm}$ due to partial screening. However, the injected quantum well carrier density is not large enough to completely screen the built-in field, which is in agreement with our previous investigation of edge-emitting lasers [6]. This can be easily checked by converting the interface charge densities given in Table I into a uniform quantum well carrier density of $2.4 \times 10^{19} \mathrm{~cm}^{-3}$ needed for full screening. Our calculations show that current densities of more than $100 \mathrm{kA} / \mathrm{cm}^{2}$ are required for complete screening of the quantum-well polarization in our device, since many carriers recombine outside the quantum wells.

Fig. 3 also illustrates the carrier accumulation at the AlGaN stopper layer. This layer is intended to prevent electrons from leaking into the $\mathrm{p}-\mathrm{GaN}$ layer by creating an energy barrier in the conduction band. The energy barrier is more than $200 \mathrm{meV}$ high without polarization (dashed line in Fig. 2). With full polarization, it is substantially reduced by the high density of positive polarization charges at the InGaN-AlGaN interface, which attract a high electron density and lead to strong band bending.

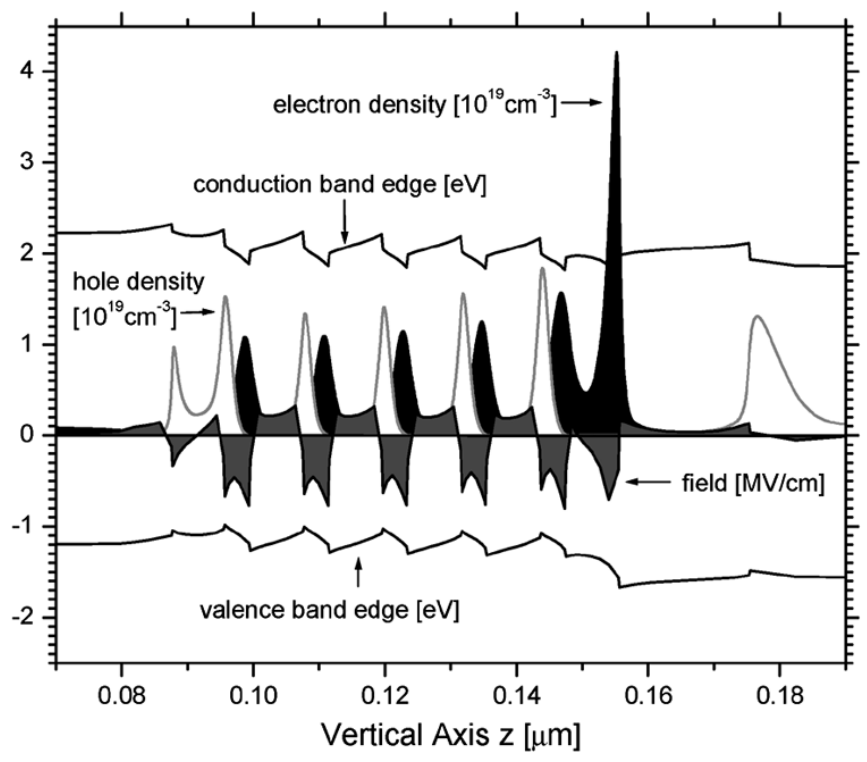

Fig. 3. Vertical profile of carrier densities and internal electrostatic field for full polarization and $j=10 \mathrm{kA} / \mathrm{cm}^{2}$ (same band diagram as in Fig. 2, AlGaN hole density $=10^{17} \mathrm{~cm}^{-3}$ ).

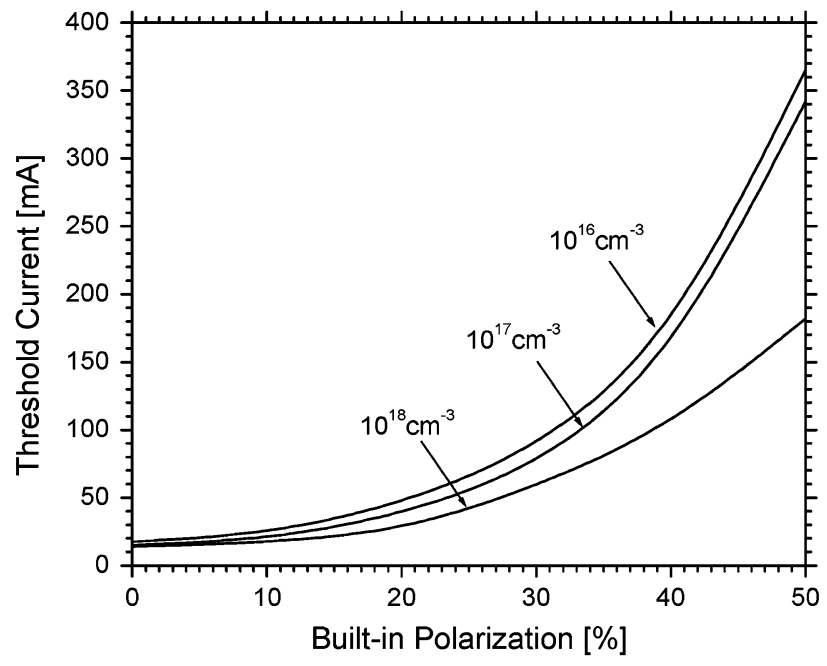

Fig. 4. VCSEL threshold current versus built-in polarization charges with AlGaN hole density as a parameter (100\% polarization corresponds to data given in Table I).

The corresponding increase in electron leackage is expected to have detrimental effects on the VCSEL threshold current.

Experimental investigations of similar quantum wells often give weaker built-in fields than predicted, ranging from $20 \%$ [11] to $80 \%$ [12] of the theoretical value, with typical results near 50\% [13]. This broad variation has been attributed to partial compensation of the built-in polarization by fixed defect and interface charges [14] or to inappropriate analysis of measured data [15]. On the other hand, the theoretical polarization model may deviate from reality, especially for InGaN, as only AlGaN measurements have been used for validation [1]. Since the actual polarization in our device is unknown, we are using the magnitude of the polarization charges as a parameter in the following.

Fig. 4 shows the calculated VCSEL threshold current as a function of polarization strength. Without polarization, the 


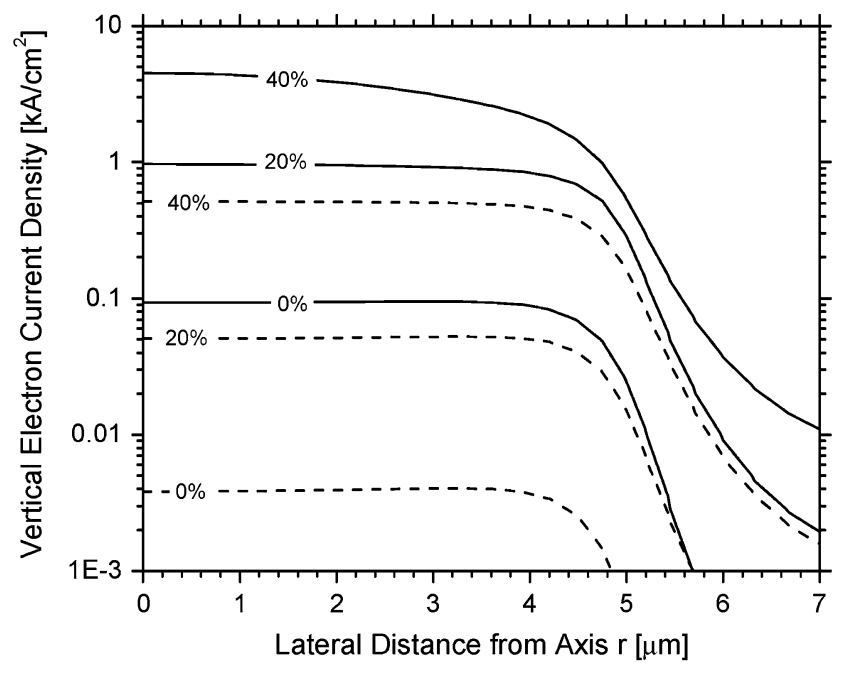

Fig. 5. Lateral profile of electron leakage at AlGaN-GaN interface for different polarization charges and $\mathrm{AlGaN}$ hole densities. Solid line is $10^{17} \mathrm{~cm}^{-3}$ and dashed line is $10^{18} \mathrm{~cm}^{-3}(100 \%$ polarization corresponds to data in Table I).

threshold current is about $15 \mathrm{~mA}$ and it rises by more than one order of magnitude for only half the theoretically predicted polarization strength. This is mainly attributed to the increasing carrier separation in the quantum wells but also to the increasing electron leakage [9], which is known to depend on the p-doping of the stopper layer [16]. In our case [8], the $\mathrm{AlGaN}$ hole density is on the order of $10^{17} \mathrm{~cm}^{-3}$. Fig. 4 shows that increasing the AlGaN hole density substantially lowers the VCSEL threshold current. This is further illustrated in Fig. 5, which compares the lateral profiles of the electron leakage current above the stopper layer. Only $20 \%$ polarization results in a dramatic increase of the electron leakage by more than an order of magnitude. Almost half of all electrons leak out of the MQW at $40 \%$ polarization and $10^{17} \mathrm{~cm}^{-3}$ hole density. Increasing the AlGaN hole density to $10^{18} \mathrm{~cm}^{-3}$ leads to a reduction of the electron leakage by about one order of magnitude. This decline in electron overflow is attributed to a higher AlGaN energy barrier relative to the electron quasi-Fermi level. Electron leakage effects are also indicated by the measured photoluminescence spectrum of our VCSEL [8] as well as by investigations on similar devices [17].

In conclusion, we have demonstrated that built-in polarization is one of the main obstacles that prevent c-plane InGaN-GaN VCSELs from lasing. Contrary to common belief, the quantumwell polarization is hardly screened at the high current densities typical for the operation of GaN-based laser diodes. The often neglected polarization of the AlGaN stopper layer is found to enhance electron leakage dramatically causing an additional increase of the threshold current. Significant reduction of the threshold current should be achieved by growing the epitaxial structure along another crystal orientation with less built-in polarization [18].

\section{REFERENCES}

[1] V. Fiorentini, F. Bernardini, and O. Ambacher, "Evidence for nonlinear macroscopic polarization in III-V nitride alloy heterostructures," Appl. Phys. Lett., vol. 80, p. 1204, 2002.

[2] L. H. Peng, C. W. Chuang, and L. H. Lou, "Piezoelectric effects on the optical properties of strained InGaN quantum wells," Appl. Phys. Lett., vol. 74, pp. 795-797, 1999.

[3] F. Della Sala, A. Di Carlo, P. Lugli, F. Bernardini, V. Fiorentini, R. Scholz, and J.-M. Jancu, "Free-carrier screening of polarization fields in wurtzite GaN/InGaN laser structures," Appl. Phys. Lett., vol. 74, pp. 2002-2004, 1999.

[4] PICS3D by Crosslight Software, 2005.

[5] J. Piprek, Semiconductor Optoelectronic Devices: Introduction to Physics and Simulation. San Diego, CA: Academic, 2003.

[6] J. Piprek and S. Nakamura, "Physics of high-power InGaN/GaN lasers," in Inst. Elec. Eng. Proc. Optoelectron., vol. 149, 2002, p. 145.

[7] A. V. Nurmikko and J. Han, "Progress in blue and near-ultraviolet vertical-cavity emitters: A status report," in Vertical-Cavity Surface-Emitting Laser Device, H. Li and K. Iga, Eds. Berlin, Germany: Springer, 2003.

[8] T. Margalith, "Development of growth and fabrication technology for gallium nitride-based vertical-cavity surface-emitting lasers," Ph.D. dissertation, Materials Dept., Univ. California, Santa Barbara, 2002.

[9] K. Domen, R. Soejima, A. Kuramata, and T. Tanahashi, "Electron overflow to the AlGaN p-cladding layer in InGaN/GaN/AlGaN MQW laser diodes," MRS Internet J. Nitride Semicond. Res., vol. 3, no. 2, 1998.

[10] J. Piprek, R. Farrell, S. DenBaars, and S. Nakamura, "Analysis of InGAN/GaN VCSELs," in Proc. 5th IEEE/LEOS Int. Conf. Numerical Simulation of Optoelectronic Devices (NUSOD), Berlin, Germany, 2005.

[11] S. F. Chichibu, A. C. Abare, M. S. Minsky, S. Keller, S. B. Fleischer, J. E. Bowers, E. Hu, U. K. Mishra, L. A. Coldren, S. P. DenBaars, and T. Sota, "Effective bandgap inhomogeneity and piezoelectric field in InGaN/GaN multiquantum well structures," Appl. Phys. Lett., vol. 73 , pp. 2006-2008, 1998.

[12] F. Renner, P. Kiesel, G. H. Döhler, M. Kneissl, C. G. Van de Walle, and N. M. Johnson, "Quantitative analysis of the polarization fields and absorption changes in InGaN/GaN quantum wells with electroabsorption spectroscopy," Appl. Phys. Lett., vol. 81, pp. 490-492, 2002.

[13] H. Zhang, E. J. Miller, E. T. Yu, C. Poblenz, and J. S. Speck, "Measurement of polarization charge and conduction-band offset at InGaN/GaN heterojunction interfaces," Appl. Phys. Lett., vol. 84, pp. 4644-4646, 2004.

[14] J. P. Ibbetson, P. T. Fini, K. D. Ness, S. P. DenBaars, J. S. Speck, and U. K. Mishra, "Polarization effects, surface states, and the source of electrons in AlGaN/GaN heterostructure field effect transistors," Appl. Phys. Lett., vol. 77, pp. 250-252, 2000.

[15] I. H. Brown, I. A. Pope, P. M. Smowton, P. Blood, J. D. Thomson, W. W. Chow, D. P. Bour, and M. Kneissl, "Determination of the piezoelectric field in InGaN quantum wells," Appl. Phys. Lett., vol. 86, p. 131108 , 2005 .

[16] R. F. Kazarinov and M. R. Pinto, "Carrier transport in laser heterostructures," IEEE J. Quantum Electron., vol. 30, no. 1, pp. 49-53, Jan. 1994.

[17] J. Piprek and S. Li, "GaN-based light emitting diodes," Optoelectronic Devices: Advanced Simulation and Analysis, 2005.

[18] S. H. Park, "Crystal orientation effects on electronic properties of wurtzite InGaN/GaN quantum wells," J. Appl. Phys., vol. 91, p. 9904, 2002 . 